\title{
De Bob Denard aux sociétés militaires privées à la française
}

Philippe Chapleau

\section{(2) OpenEdition}

\section{Journals}

Édition électronique

URL : http://journals.openedition.org/conflits/977

DOI : $10.4000 /$ conflits.977

ISSN : $1777-5345$

Éditeur :

CCLS - Centre d'études sur les conflits lilberté et sécurité, L'Harmattan

Édition imprimée

Date de publication : 1 décembre 2003

Pagination : 49-66

ISBN : 2-7475-6065-1

ISSN : 1157-996X

Référence électronique

Philippe Chapleau, « De Bob Denard aux sociétés militaires privées à la française », Cultures \& Conflits

[En ligne], 52 I hiver 2003, mis en ligne le 03 juillet 2004, consulté le 30 mars 2021. URL : http:// journals.openedition.org/conflits/977 ; DOI : https://doi.org/10.4000/conflits.977

Ce document a été généré automatiquement le 30 mars 2021.

Creative Commons License 


\title{
De Bob Denard aux sociétés militaires privées à la française
}

\author{
Philippe Chapleau
}

Peut-on parler de «sociétés militaires privées françaises"? A l'heure où les prestataires de services militaires américains, britanniques et sud-africains affichent une incroyable prospérité et raflent, sur le seul marché moyen-oriental, des contrats d'une valeur totale de près de quatre milliards de dollars pour l'année 2004, l'ambition affichée par certaines entreprises françaises de se mesurer aux PMC (Private Military Companies) anglo-saxonnes, d'une part, incline à la perplexité et, d'autre part, fait s'interroger sur leurs capacités, la qualité de leurs services et leurs chances dans un environnement dominé par des sociétés comme Vinnell, DynCorp, MPRI, Sandline, Kroll, Task, Cubic, AirScan...

Les sociétés françaises sont-elles en mesure de proposer, comme l'affirment, par exemple, les dirigeants de Secopex, "une alternative au savoir-faire anglo-saxon représenté par les multiples SMP (sociétés militaires privées) américaines, britanniques ou sudafricaines ? $»^{1}$ ? Ne faut-il pas plutôt leur reconnaître des compétences particulières et un positionnement de circonstances dans des niches où elles tentent de tirer leur épingle du jeu? En fait, ces sociétés, aussi compétentes et ambitieuses qu'elles soient, doivent compter avec un environnement juridique et légal propre à la France, avec un milieu qui s'émancipe difficilement de la tradition mercenariale et une concurrence étrangère galopante.

Des SMP françaises? On va le voir, la réponse s'inscrit, une fois de plus, dans le cadre d'une «diversité quasi culturelle». Les «Corporate Warriors » américains ${ }^{2}$ n'ont pas grand-chose à voir avec leurs homologues (concurrents?) français. Mais ils ne peuvent ignorer ni la détermination ni la valeur des entreprises hexagonales positionnées sur certains créneaux, comme l'intelligence économique, le déminage ou encore le conseil. Pas plus qu'ils ne peuvent se passer des compétences des "privés " français; la réputation et la grande expérience de ces anciens soldats en font des recrues de choix pour les PMC anglo-saxonnes ${ }^{3}$.

Les années 1990 : l'ombre des mercenaires 


\section{Le crépuscule des soldats de fortune}

On peut difficilement analyser l'émergence des sociétés de services militaires ou sécuritaires françaises sans dresser un état des lieux du milieu de la décennie 1990. Depuis une trentaine d'années, les mercenaires faisaient de nouveau partie du paysage militaire mondial. Ils avaient profité des avatars de la décolonisation et de la « drôle de guerre froide» pour reprendre du service et ajouter quelques pages, souvent peu glorieuses, à leur histoire séculaire. C'était l'époque des Schramme, Tavernier, Hoare et autres Denard.

Réprimées par les Nations unies et l'Organisation de l'unité africaine en 1977 puis en 1989, les activités des «affreux » avaient également été entravées par des faiblesses intrinsèques au milieu des soldats de fortune européens. D'une part, et les mémoires des chefs mercenaires en témoignent ${ }^{4}$, le recrutement des mercenaires de l'aprèsguerre était pour le moins aléatoire. Du Katanga de 1960 aux Comores de 1995, en passant par le Biafra de 1968, il n'est pas rare de retrouver les mêmes baroudeurs vieillissants, les mêmes officiers de réserve issus du contingent, les mêmes ex-sousofficiers des paras commandos belges ou de la Légion étrangère. Des hommes à la formation et à l'expérience parfois réduites, ou encore recrutés du fait de leurs sympathies idéologiques... D'autre part, ces «intérimaires du coup de feu» ne disposaient souvent ni des moyens logistiques nécessaires à la conduite d'opérations d'envergure ni des fonds permettant un recrutement optimal et l'acquisition d'équipements adaptés. Toutes ces faiblesses conjuguées expliquent la piètre qualité de nombreuses « prestations » et la réputation scabreuse des mercenaires.

Conscient des faiblesses du milieu, le Français Bob Denard avait profité de la création, en 1978, de la garde présidentielle comorienne, pour se doter d'une base arrière permanente, d'un noyau stable de mercenaires en uniforme et d'un "cash-flow» approprié. La garde, équipée et financée par l'armée sud-africaine, était encadrée par une trentaine de mercenaires francophones. Parallèlement, Denard avait créé une société de services (gardiennage et transport) dont les contrats avec la chaîne hôtelière Sun International garantissaient la viabilité. La " petite entreprise » du chef mercenaire français lui a permis de tenir onze ans et de mettre sur pied plusieurs opérations périphériques (Tchad en 1981-1982, Vanuatu prévue pour 1990). L'expérience comorienne de Bob Denard a tourné court au moment où les Anglo-Saxons structuraient le milieu du mercenariat traditionnel et créaient les premières grandes sociétés militaires privées.

Ces entreprises allaient bénéficier de deux (r)évolutions militaires. Sur fond de pacification des tensions mondiales, les grandes armées ont massivement démobilisé. Des milliers d'hommes, formés, expérimentés mais sans grand avenir professionnel civil, se sont tournés vers l'industrie de la sécurité et vers des sociétés comme les britanniques DSL et Sandline ou comme la sud-africaine Executive Outcomes dirigée par d'anciens cadres du 32e bataillon, l'une des unités d'élite du régime blanc. Les grandes nations ont également commencé à réduire leurs budgets militaires et à pratiquer l'externalisation, voire à privatiser des missions jusque-là traditionnellement du ressort des forces armées nationales: formation, soutien, transport, déminage, sécurité des sites et des troupes, combat même... Les PMC anglo-saxonnes se sont donc engouffrées dans un prometteur marché intérieur et ont poursuivi leur développement, à l'inverse des mercenaires français, ou encore israéliens, qui ont dû se 
structurer, tout en restant cantonnés à des créneaux d'intervention beaucoup plus réduits.

Un inventaire des entreprises françaises en activité au cours des années 1990 montre trois pôles structurant : un pôle d'entreprises dirigées par d'anciens militaires, un pôle de petites sociétés liées à des mouvements extrémistes et un pôle plus traditionnel, celui de la sécurité et du gardiennage.

\section{La « GIGN Connection »}

Parmi les premiers à s'être investis dans la fourniture de services sécuritaires figure un groupe d'anciens militaires français. Des gendarmes pour la plupart. «GIGN Connection », en quelque sorte! Certains de ces hommes ont longtemps été liés aux services secrets français; quelques uns s'en sont émancipés pour proposer leurs services à de grandes entreprises ou à des États africains et moyen-orientaux. Quelques trajectoires en guise d'exemples.

Paul Barril, dont les employés ont fait parler d'eux à l'automne 2002, lors de la crise en République centrafricaine ${ }^{5}$, représente parfaitement ce type de militaire reconverti dans l'entreprenariat sécuritaire. L'ancien gendarme a créé le Groupe Barril Sécurité (www.barril.com) après une belle carrière au sein du GIGN. Le Groupe Barril Sécurité fédère plusieurs sociétés: Barril Investigation, Protection Conseil Sécurité, Groupe privé Barril, Activités sécuritaires, Security Action Store, "Haute protection " (une lettre d'information)... Le Groupe Barril Sécurité (« Un nom unique, des compétences multiples») propose plusieurs types de prestations: audit et détection d'écoutes, protection rapprochée, investigations, ingénierie, gestion de crise (parmi les crises, Paul Barril cite «campagne médiatique de médisance, chantage, menaces, séquestration, disparition, enlèvement, problèmes judiciaires, risques terroristes, formation et recrutement... »).

Philippe Legorjus est aussi un ancien gendarme. Recruté en 1978, il est affecté à l'escadron parachutiste de Mont-de-Marsan. Quatre ans plus tard, l'officier sorti major de l'école de Melun rejoint le GIGN. Après l'affaire de la grotte d'Ouvéa, Legorjus quitte la gendarmerie et entame sa reconversion dans la sécurité. Il sera conseiller du Comité olympique pour les problèmes de sécurité lors des Jeux d'Albertville. Il conseillera Christian Blanc, alors PDG d'Air France. En 1993, Philippe Legorjus crée une petite société : PHL Consultants, qui propose d'« accompagner les entreprises françaises dans les zones à risques pour sécuriser leurs opérations logistiques et financières à l'export ». Dans la liste de ses clients figure Total. L'entreprise est présente en Birmanie et ses opérations sont alors menacées par la guérilla Karen (que soutiennent d'autres petites structures françaises et qui comptent dans leurs rangs des mercenaires français). En 1995, il fonde Atlantic Intelligence (www.atlantic-intelligence.fr), une entreprise spécialisée dans l'intelligence économique. La société qui dispose de bureaux à Nantes et à Paris, offre des prestations dans six domaines: conseil en veille et intelligence économique, recherche d'informations, audit, appui au développement et lobbying, gestion et communication de crise et ingénierie de sûreté et de sécurité. Depuis novembre 2001, Atlantic Intelligence est présente sur le marché libre de la Bourse de Paris. AI dispose d'un portefeuille de plus de 150 entreprises (dont une douzaine du Cac 40, comme Total, Sodexho, Bouygues, Danone, Axa). Des projets d'expansion vers l'Europe de l'Est et l'Amérique du Sud ont été envisagés. En août 2003, Atlantic Intelligence et le Stirling Group ont créé Stirling Atlantic, une joint-venture spécialisée dans la gestion des risques internationaux. 
Robert Montoya s'est aussi reconverti dans la sécurité après son passage dans les rangs de la gendarmerie. Il a été très actif en Afrique : au Togo (entraînement et équipement des forces anti-émeutes du régime du général Eyadéma), au Zaïre et au Gabon en particulier, au travers du groupe SAS (Security Advisory and Service), dont un des premiers clients a été le transporteur Géodis-Calberson. Reconnu «comme l'un des principaux acteurs de la sécurité et de logistique africaine ", Robert Montoya dirige aussi Darkwood Logistique, une société de transport installée au Togo. Aujourd'hui, Robert Montoya dirigerait plus de 2500 personnes sur sept pays et propose des missions de conseil et d'audit, de sécurité des personnes et des biens, de sûreté aéroportuaire, de sûreté des compagnies aériennes, d'accompagnement à l'export, de renseignement économique...

Jean-Louis Chanas appartient lui aussi à la cohorte des anciens. Ce camarade de promotion de l'ex-gendarme élyséen Christian Prouteau serait « l'un des anciens de la DGSE, qui a le mieux réussi » sa reconversion dans le privé. Eric Sa a été présentée, dans un article de La Tribune de juillet 2001, comme "l'une des plus anciennes entreprises d'intelligence économique et l'une des cinq principales » et " férocement alignée par le directeur des renseignements généraux de la préfecture de police ». Effectivement, le torchon a brûlé, en 1999, entre Jean-Pierre Pochon, le directeur des RG à la préfecture de police de Paris, et Eric Sa. En fait, dans sa déposition devant la commission d'enquête parlementaire sur le DPS, le service d'ordre du Front national, Jean-Pierre Pochon évoquait la société de Jean-Louis Chanas et déclarait que Eric Sa «coiffe un réseau de mercenaires actif dans les milieux d'extrême droite ».

La mouvance néofasciste

Effectivement, Eric Sa a fait partie d'un groupe de sociétés mises sous surveillance à la fin des années 90 par les services de police.

Selon Jean-Pierre Pochon (son témoignage date du 2 mars 1999), "des liens étroits existent parfois» entre le DPS et certaines sociétés de sécurité. "Quelques sociétés de sécurité sont directement gérées par des militants du Front national (...) Toutes ces sociétés amies peuvent à l'occasion épauler le DPS en lui fournissant du matériel(...).En fait, il existe une grande perméabilité entre le DPS, certaines sociétés de sécurité et des réseaux mercenaires ».

L'enquête parlementaire a fait émerger certains noms de sociétés et d'individus. Le Réseau Voltaire a publié la liste des " principales sociétés de sécurité » proches du DPS (ACDS, Ambassy, Bègue Consultants, Eric Sa, Groupe Onze International devenu ILS, Normandy, OGS, SPGM) et dans sa série de portraits de membres des services d'ordre du FN ou du MNR figurent de nombreux mercenaires ou anciens militaires dirigeants de sociétés de sécurité actives en Afrique ${ }^{6}$. Aucune des sociétés soupçonnées de liens avec l'extrême droite ou placées sous surveillance par la police n'avait, en tout cas, l'envergure d'une PMC. Ces entreprises constituaient, tout au plus, des «salles d'attente » pour des recrutements limités de mercenaires ou des contrats ponctuels de sécurité ou d'assistance militaire en Afrique. En outre, leurs moyens humains, financiers et matériels étaient beaucoup trop limités pour qu'elles puissent, à l'époque, prétendre se poser en véritables prestataires de services pour le ministère de la Défense si celui-ci avait décidé de privatiser certaines missions.

L'industrie de la sécurité

Troisième pôle: l'industrie de la sécurité où se sont reconvertis de nombreux démobilisés des années 1990 et qui a permis à des entreprises tentées par les prestations à l'international de décrocher, sur le marché intérieur, des «contrats de 
subsistance ». Ce secteur économique était alors, et est toujours, remarquablement dynamique, même en France ${ }^{7}$.

L'industrie de la sécurité pourrait en fait constituer un vrai terreau pour les " graines » de PMC françaises. Le nombre grandissant d'entreprises illustre parfaitement la croissance de la demande pour des services de protection et de gardiennage. Certes, l'industrie de la sécurité n'a pas toujours eu une bonne image. Dirigeants peu scrupuleux, personnels sans réelles qualifications, liens occultes avec des partis politiques ou des mouvements paramilitaires : les accusations ne manquent pas. Mais le secteur se professionnalise, s'assainit et se dynamise, en particulier avec l'arrivée de sociétés étrangères sur le marché hexagonal ${ }^{8}$. Exemple avec la société danoise Group 4 Falck.

Le Group 4 Falck est en train de réaliser une percée mondiale; il emploie plus de 230 000 personnes dans 80 pays. Créée en 1901 pour Group 4 et 1906 pour Falck, l'entreprise danoise s'est implantée en Grande-Bretagne en 1950 ; entre 1980 et 1999, elle a procédé à de nombreuses acquisitions en Europe, en Asie et en Amérique. La fusion des deux entités a eu lieu en 2000 , année de la première acquisition d'une entreprise française : Securicor France (gardiennage et transports de fonds). En février 2001, Group 4 Falck procède au rachat d'Euroguard, le numéro deux de la profession derrière Securitas. En mai de la même année, OGS (Omnium de gardiennage et sécurité) était rachetée. C'était, selon les spécialistes, l'une des rares sociétés de surveillance dont le chiffre d'affaires dépassait les cent millions de francs (15 millions d'euros) et qui était encore indépendante. En janvier 2002, Group 4 Falck achète Cobelguard. Désormais, le groupe danois emploie plus de 7000 personnes en France pour un chiffre d'affaires de plus de 183 millions d'euros. Ses activités sont regroupées au sein de six filiales: Euroguard (surveillance humaine et industrielle, sûreté aéroportuaire), Eurovaleurs (transports de fonds), Vardel (sécurité électronique), SGO (Société de gardes opérationnels pour la protection rapprochée), Euroguard Service (prestations multiservices non sécuritaires) et Iffis (institut français de formation et d'ingénierie sécuritaire). Group 4 Falck (www.group4falck.com et www.euroguard.fr), qui est spécialisé dans les prestations de concessions de projets publics, pourrait se positionner sur le marché de la prestation militaire (comme il le fait aux États-Unis depuis son rachat de Wackenhut) si la Défense française décidait d'externaliser davantage et de recourir au privé.

Au cours des années 1990, les prestations offertes par les entreprises françaises sont variées. Elles vont de la protection rapprochée (Groupe Barril) à la formation (SAS en Afrique), en passant par la gestion du risque (PHL en Asie), l'intelligence économique (Atlantic Intelligence), la sécurisation des sites (SIA, Société internationale d'assistance, en Angola, OGS en Birmanie), le déminage (Pretory Technologie en Bosnie)... Les clients sont majoritairement des entreprises (Elf, Bouygues et Total figurant parmi les plus importantes) dont une partie des activités sont localisées dans des zones à risques (Afrique et Asie principalement). Les prestations, confiées à d'anciens soldats mais sans franche connotation militaire, restent cependant aléatoires et ponctuelles. Elles sont soumises à la fréquentation de réseaux d'influence, au bon vouloir du gouvernement et des services secrets français qui recourent aux services de certaines de ces sociétés notamment dans le "pré carré africain ». Nulle surprise, dès lors, qu'à cette époque de structuration entrepreneuriale, on ait assisté, en parallèle, à des opérations classiques montées et effectuées par des mercenaires free-lance agissant en " corsaires ", ou tout du moins avec le fameux «feu orange » dont parlait Bob Denard lors de son procès de 
1994 : les plus récentes péripéties de mercenaires aux Comores, en Côte d'Ivoire et à Madagascar s'inscrivent dans ce cadre.

Des sociétés militaires privées à la française

$P M C$ : une longueur d'avance

Actuellement, le chiffre d'affaires annuel des prestataires de services militaires anglosaxons est estimé à 100 milliards de dollars. Un résultat impressionnant favorisé par quatre facteurs : des fusions de PMC, l'arrivée de sociétés traditionnellement " civiles », la diversification de leurs prestations et la privatisation de la fonction régalienne.

Les exemples de fusions et de rachats sont nombreux: DynCorp a été racheté par Computer Sciences Corporation, MPRI par L3 Communication, DSL (une entreprise britannique) par ArmorHolding (qui a créé ArmorGroup), Gray Security (PMC sudafricaine) par le britannique Securicor, Trojan par International Risks Management (également britannique), Vinnell par Northrop Grumman, Wackenhut par le Group 4 Falck, Betac par ACS Defense Inc., Vance par SPX Corporation, Titan par Lockheed Martin, Crucible par Kroll... La plupart de ces entreprises sont cotées en Bourse et certaines emploient des dizaines de milliers de salariés.

A ces sociétés s'ajoutent désormais des entreprises de génie civil (Kellog Brown and Root, Pacific and Engineers, Parsons, Perini), des logisticiens (ITT, Combat Support and Associates, Artic Slope World Service), des sociétés aériennes (Airscan, ICI, SkyLink Air), des entreprises spécialisées dans la formation (Cubic, Betac, Kroll)... Toutes ces entreprises ont mis sur pied des filiales spécialisées dans la sécurité et les prestations militaires et profitent du grand marché de la privatisation de la défense et de la création, suite aux attaques terroristes du 11 septembre 2001, de la création du Homeland Security Department. Entre 1994 et 2002, le ministère américain de la Défense a passé plus de 3000 contrats avec des PMC, pour une valeur de plus de 300 milliards de dollars. Le Pentagone (mais aussi le Département d'État) confie désormais la gestion de ses bases, l'entretien de ses équipements, la formation initiale et l'entraînement des troupes, l'écriture de la doctrine, le transport, la sécurité de ses sites et de ses unités, le renseignement aux «Corporate Warriors » de ces nouvelles ANG (armées non gouvernementales) qui épaulent et parfois remplacent les GI's lors de certaines opérations extérieures. Ainsi en Irak où opèrent les Américains de Vinnell, MPRI, DynCorp, SAIC, ArmorGroup, Kroll, Custer Battles, Guardsmark..., les Britanniques de Control Risks, Hart Group, Global Risk, ISEC, Olive Security, Securicor, Pilgrims, Genric, Janusia, les Sud-Africains de Meteoric Tactical Solutions et Erinys ou encore les Danois de Group 4 Falck ${ }^{9}$. Au total, une soixantaine d'entreprises.

Défense française : la « res publica »

Les caractéristiques distinctives des PMC anglo-saxonnes contemporaines s'appliquent difficilement aux sociétés françaises. Celles-ci restent des structures de petite taille, assez spécialisées, opérant dans un créneau réduit où les grands prestataires de services civils n'ont ni ambition ni intérêts. En outre, et plus que tout, elles doivent compter avec la frilosité chronique des autorités françaises à externaliser des activités militaires majeures. Le ministère de la Défense consent, tout au plus, à confier à des entreprises privées l'entretien des espaces verts, le transport des personnels et l'entretien de la bureautique ${ }^{10} .$. Pour 2004, le gouvernement a cependant annoncé qu'il va confier « à des sociétés privées la gestion des logements des gendarmes et de 25000 véhicules civils, ainsi que la fourniture d'heures de vol pour la formation initiale des pilotes 
d'hélicoptère ${ }^{11}$. On est cependant bien loin des missions confiées aux PMC par les Américains, les Britanniques, les Sud-Africains...

Seule exception apparemment notable, Défense Conseil International (www.groupedci.com). Cette société de droit privé, sous tutelle du ministère de la Défense, regroupe plus de 800 personnes : militaires détachés et retraités des armées et de la DGA, qui remplissent des missions de formation des armées, de conseil auprès des états-majors et des organismes gouvernementaux. DCI se présente comme le "spécialiste de la formation, du conseil et de l'assistance militaire (...) auprès des armées des pays amis dans le monde entier ». Il compte des «clients et partenaires dans plus de 30 pays». DCI dispose de six filiales. Navco, créée en 1980, offre ses services aux marines étrangères. Airco, créée en 1984, offre les mêmes prestations aux armées de l'air d'une vingtaine de pays. Desco (Défense Système Conseil), créée en 1990, supervise le déroulement des programmes d'exportation d'armement et offre des prestations de conseil et d'assistance dans le domaine de la reconversion des industries d'armement. Stratco a été créée en 1991 "afin d'élaborer une capacité de réflexion stratégique au profit des organismes de la Défense et des industriels français, ainsi qu'au profit de pays amis avec lesquels la France souhaite développer des partenariats ». L'entreprise intervient aussi " pour des prestations touchant à la géostratégie, les concepts de défense, l'environnement, l'intelligence économique et l'aide humanitaire ». Une société de capital-risque a été créée en 1992 : la Financière de Brienne, spécialisée dans le financement des PME-PMI de haute technologie. La dernière filiale a été créée en 1972. La Cofras (Compagnie française d'assistance spécialisée) assure la formation militaire technique, offre une assistance pour l'emploi des forces et assure un soutien technique auprès des clients qui ont acquis des matériels français (blindés, artillerie, hélicoptères). La Cofras a disposé ellemême d'une filiale, jusqu'en 1999, la Cidev (Conseil international et Développement), créée après la guerre du Golfe et chargée de conduire des opérations de déminage humanitaire avec des financements nationaux, européens ou internationaux. La Cidev est ainsi intervenue au Cambodge (sur un contrat européen), au Mozambique (avec des fonds de l'Agence française de développement) et en Angola (une opération subventionnée par le ministère de la Coopération).

DCI est certes un groupe de droit privé mais l'État détient 49,9\% des actions. Les 50,1\% restants sont entre les mains des l'office général de l'Air, la Sofresa (Société française d'exportation de systèmes d'armements) et l'ofema (Office français d'exportation de matériels aéronautiques), trois offices publiques d'armement. Ses activités illustrent, pour reprendre les mots de Belkacem Elomari, directeur de l'Observatoire des transferts d'armements, "une forme de privatisation de la coopération militaire française $»^{12}$. Une privatisation très encadrée, financièrement d'une part, et humainement d'autre part, puisque l'État « peut s'assurer la subordination » des personnels, militaires ou exmilitaires, de DCI et que tous les dirigeants de la société sont nommés par le gouvernement français. «Bras séculier des états-majors » ou « auxiliaire de l'État pour les exportations d'armements ", DCI ne dispose que d'une marge d'autonomie très restreinte. Son statut de société anonyme ne doit pas leurrer : DCI n'est pas encore une société commerciale indépendante mais elle pourrait trouver sa place dans l'inventaire des SMP à la française.

Des challengers français?

Cette répugnance française à privatiser la fonction régalienne détermine, et restreint, autant l'éventail des prestations que la liste des clients éventuels. Ne pouvant pas 
compter sur des contrats gouvernementaux d'importance, les sociétés françaises doivent se rabattre vers des clients commerciaux (entreprises, collectivités), des clients privés (hommes d'affaires, diplomates, VIP) et tenter de décrocher des contrats ponctuels à l'international auprès d'ONG opérant en zones à risques ou de gouvernements de pays en développement.

Alors que la nomenclature des services offerts par les PMC anglo-saxonnes s'organise autour de trois pôles d'activités : soutien, conseil et projection opérationnelle, à forte connotation militaire ${ }^{13}$, les sociétés françaises disposent d'un "catalogue " plus restreint, nettement moins militarisé et, généralement, sans rubrique "Soutien » (transport, génie civil, maintenance).

La sûreté (sécurité des personnes et des biens, protection rapprochée, aussi bien en France qu'à l'étranger) constitue un premier créneau où se sont positionnées de très nombreuses entreprises: Atlantic Intelligence (en partenariat avec le groupe Stirling. $36 \%$ du chiffre d'affaires d'AI provient de la sûreté), Géos (15\% de son chiffre d'affaires), Group 4 Falck (avec sa filiale SGO), le groupe Alliance Prestige (avec sa filiale AEPR), Defense Control (une société de droit luxembourgeois), Sécurité sans Frontières (SSF, filiale de Mobility Saint-Honoré, elle-même filiale du Groupe Assurances et Conseils Saint-Honoré qui s'intéresse de près au domaine "de la sécurité et de la sûreté des personnes » et a embauché le général Costedoat, ex-patron du service Action de la DGSE), le groupe Barril Sécurité... Quelques remarques : c'est, d'abord, un domaine où se recyclent de nombreux anciens militaires et policiers, une véritable " antichambre » pour des professionnels en attente de contrats plus adaptés à leurs qualifications et mieux rétribués; c'est aussi un domaine actif ${ }^{14}$ qu'aucune SMP ne peut négliger puisqu'il génère des recettes assez stables; c'est, enfin, un domaine chroniquement fragile où $48 \%$ des 1650 entreprises recensées par le Syndicat national des entreprises de sécurité emploient moins de 5 salariés, $25 \%$ entre 5 et 19 et $19 \%$ entre 19 et 99 !

Autre créneau : l'intelligence économique. Une niche où de nombreux cabinets tentent de se faire une petite place. Cette «myriade de petites structures » françaises où, selon La Tribune, "règne l'opacité, générée par la culture des praticiens, souvent issus des services ou de la police $»^{15}$, a fort à faire face à la concurrence des Kroll et autres Control Risks. Et pourtant, les entreprises françaises relèvent le défi. L'intelligence économique permet à Géos de générer le quart de ses 12,7 millions d'euros de chiffre d'affaires; la société i2f (Groupe Assurances et Conseils Saint-Honoré) a racheté Circé (une société créée par un ancien colonel de gendarmerie et spécialisée dans l'IE); Salamandre (où l'on retrouve l'ancien directeur de la DGSE, le général Mermet) et sa société sœur SAS (créée par deux anciens hauts fonctionnaires de la police nationale) s'imposent dans l'IE hightech... Autour de ces sociétés gravitent Atlantic Intelligence (1er cabinet d'IE coté en Bourse), BD Consultants, C4iFR (dont l'un des dirigeants est Christian Harbulot, le directeur de l'École de guerre économique), AB Associate, etc.; au total, une bonne vingtaine de sociétés qui tentent d'atteindre une taille critique et doivent compter avec la forte concurrence anglo-saxonne.

Ces sociétés spécialisées dans l'intelligence économique s'intéressent souvent à la gestion du risque. Avec la multiplication des zones sensibles, ce marché croît de près de $10 \%$ par an. On retrouve sur ce créneau les Atlantic Intelligence, Géos (qui offre à ses abonnés un service spécialisé : Géos crise24), Sécurité sans Frontières (qui propose des audits sûreté-sécurité) ou encore Defense Control. 
Autre créneau sur lequel les sociétés françaises proposent leurs services : la formation et le conseil. L'éventail est large : sensibilisation des personnels avec Géos et SSF, tir, protection rapprochée et mise en situation extrême avec CITS (une filiale du groupe Alliance Prestige) et le groupe Barril Sécurité. Plusieurs entreprises proposent, par ailleurs, de la formation et de l'instruction militaires : instruction au tir, entraînement d'unités spécialisées, techniques d'intervention. SAS, la société de Robert Montoya, a ainsi entraîné des forces anti-émeutes togolaises. Mais ce type de prestations est évidemment très encadré et rarement sous-traité par le ministère de la Défense.

Dernier secteur où des entreprises françaises sont actives et où d'éventuelles sociétés militaires privées «à la française " pourraient s'impliquer : le déminage humanitaire, qui nécessite des connaissances et une expérience que, seul, un passage dans les forces armées permettent d'acquérir. On l'a vu, l'ex-Cidev, Conseil international et Développement, a déjà effectué des opérations de déminage humanitaire. Parmi les sociétés présentes sur ce créneau, citons Europérations, active depuis 2003 au Cambodge (dans le cadre, semble-t-il, d'un accord pour déminer une région abritant des mines de saphirs et de rubis), Hamap (Halte aux mines antipersonnels, une association qui maintient "une équipe-action permanente composée de membres qui ont une longue expérience du déminage»), la Compagnie française de déminage (dont les principaux actionnaires seraient la société de sécurité Alliance Prestige et la SNGTS). Citons aussi IIC (International Instruction Corps) qui se présente comme "une société d'instruction dans les domaines de la sécurité, de la formation militaire et du déminage ». Cette société franco-espagnole a, selon l'un de ses dirigeants, effectué des missions de déminage au Moyen-Orient, en Centrafrique et dispensé de la formation en Mauritanie. Le poids lourd du secteur reste Géomines. Créé en 1995, lors des opérations de déminage du port de Beyrouth, Géomines appartient au groupe Géocéan. Cette société spécialisée dans le déminage terrestre et sous-marin est intervenue en Bosnie, au Cambodge, en Irak, à Taïwan, au Maroc... Géomines est également intervenue en Irak, au début de l'année 2004, dans le cadre d'un contrat de sous-traitance pour la PMC américaine Steele Foundation. Un de ses démineurs ayant été tué dans une embuscade, la société française a pris la décision de se retirer d'Irak.

Des challengers qui partent de loin

Deux petites sociétés françaises affichent leur ambition de concurrencer les PMC anglosaxons traditionnelles sur deux de leurs trois pôles d'activité majeurs : le conseil et la projection opérationnelle. Le positionnement de ces challengers illustre parfaitement les limites du secteur français dans son ensemble.

Le groupe Earthwind Holding Corporation (EHC) a été créé en 1999. Cette société basée au Luxembourg tente de " coller » aux modèles anglo-saxons. Une simple visite sur son site Internet (www.groupe-ehc.com) permet de mesurer cette concordance d'identité, de prestations et même d'éthique. EHC se présente comme "la seule société francophone réunissant sous la même enseigne des compétences en sécurité-sûreté, assistance militaire privée, support opérationnel, logistique, mise à disposition de matériels et équipements et formation ». Plus précisément, EHC propose du conseil stratégique et confidentiel, de la sécurité pour les entreprises à l'étranger, du soutien pour les institutions internationales, les ONG et les médias, de la formation, de l'instruction et du soutien pour les forces armées et, enfin, du soutien aux forces de police et aux agences de sécurité. Exactement ce que proposent les Britanniques de Sandline, Genric, Olive Security, ISEC, etc., les Américains de DynCorp, MPRI, Crucible, et des sociétés 
«multinationales» comme Erynis (anglo-sud-africaine), Presidium International (anglo-italienne, enregistrée aux Seychelles).

Parmi ses dirigeants, on retrouve d'anciens cadres des forces armées françaises. L'un d'eux a été impliqué dans plusieurs opérations de mercenaires en Afrique. Un autre cadre de EHC serait le représentant (en fait, le "Operations Manager Luxembourg ») de Northbridge Services Group, une PMC britannique ambitieuse et entreprenante, impliquée dans plusieurs projets en Afrique (imposition de la paix en RDC, capture du Libérien Charles Taylor, sécurisation au Nigeria). Northbridge, désormais en mesure de recruter du personnel francophone via EHC, est également présente aux USA depuis l'acquisition de la PMC Executive Outcome Inc (aucun lien avec la PMC sud-africaine Executive Outcomes, qui a fermé en 1998). Pour sa part, fin 2003 et début 2004, EHC a effectivement recruté pour le compte de PMC anglo-saxonnes. Ces sociétés étaient à court de personnels qualifiés pour des missions de protection en Irak où le marché connaît un boom phénoménal.

Secopex, créé en 2003, veut, d'une part, « proposer une alternative française au savoir-faire anglo-saxon représenté par les multiples SMP américaines, britanniques ou sud-africaines » et, d'autre part, "proposer des prestations globales au-delà de ce que proposent les sociétés de sécurité privée traditionnelles françaises (Géos, SSF, AI) ». Cette société a été créée en mai 2003 par d'anciens sous-officiers d'un régiment de parachutistes de l'infanterie de marine. Même si elle annonce qu'elle "ne mènera pas d'activités combattantes, ne participera pas à des conflits armées », Secopex a tout d'une PMC : son recrutement («des experts et des spécialistes issus des formations d'élite des ministères de la Défense et de l'Intérieur »), son objectif (" apporter des éléments de réponse face à une multitude de menaces aux contours mal définis: terrorisme, crise, criminalité, guerre civile» en combinant "les volets politique, militaire, sécuritaire, humanitaire et économique»), ses prestations ("support opérationnel, sécurité opérationnelle, intelligence économique, formation et conseil militaire »). D'une PMC, Secopex a aussi la « virtualité » : un site Internet (comme toutes les PMC qui soignent leur image), un discours lissé (qui sera mis à l'épreuve des faits) où dominent deux principes (l'éthique et la légitimité), un « fichier » dans quel les deux salariés de l'entreprise peuvent piocher, et, last but not least, des clients potentiels...

Les trajectoires de Secopex et EHC témoignent, en somme, de la situation périlleuse des sociétés militaires françaises. Premièrement, elles sont contraintes, soit de jouer la carte du rapprochement avec les structures anglo-saxonnes qui contrôlent l'essentiel du marché de la prestation de services militaires, soit d'afficher leur différence sur l'étroit marché hexagonal où la concurrence est déjà rude. Deuxièmement, ces SMP ne peuvent guère compter sur des rapprochements avec de grosses entreprises nationales qui pourraient injecter des capitaux et doper leurs capacités opérationnelles; les géants français de l'énergie, du BTP ou du transport ont, tout au plus, recours aux prestataires de services sécuritaires ou militaires, pour des missions ponctuelles, limitées, liées à des crises... Troisièmement, elles ne peuvent, pour l'instant en tout cas, espérer aucune «mission » d'importance de la part des ministère français de la Défense et des Affaires étrangères qui externalisent au compte-gouttes et tiennent à se démarquer des « entrepreneurs de guerre ». L'État français entend rester le dépositaire des instruments militaires et conserver le monopole du recours à la force ; la loi d'avril 2003 sur le mercenariat et les interventions militaires françaises en Côte d'Ivoire, en Ituri (RDC) et au Liberia le prouvent. Les sociétés militaires privées passent, aux yeux de l'État français, pour une « déviation » anglo-saxonne, pour un épiphénomène dont 
on refuse d'anticiper l'impact sur les relations internationales, l'éthique, l'industrie de la défense, les relations civilo-militaires, le droit international, les droits de l'homme...

Pourtant, inéluctablement, la France va devoir envisager une privatisation de sa Défense et un recours intensif au service privé. Il est donc temps de conforter les prestataires français de services militaires, de les associer aux actions gouvernementales de formation des armées étrangères, de protection des ONG, plutôt que de devoir, le jour venu, ne pouvoir faire autrement que de signer des contrats avec des firmes étrangères, au risque d'affaiblir la souveraineté nationale.

"Alternatives» aux Private military companies anglo-saxonnes? L'ambition actuelle des sociétés militaires privées françaises ne doit être ni de vouloir concurrencer les firmes américaines, britanniques et sud-africaines, ni de vouloir reprendre à leur compte les missions qui incombent aux forces armées nationales. Leur effort doit être pédagogique. Elles doivent convaincre de leur légitimité morale, de leur crédibilité professionnelle, de leur indépendance politique et de leur capacité à s'inscrire dans des actions de complémentarité avec les forces armées nationales dont le reformatage a exacerbé certaines faiblesses. Certaines de ces insuffisances « procèdent de la réduction $d u$ volume des troupes prépositionnées et des faiblesses capacitaires de nos moyens de projection ${ }^{16}$. L'insuffisance des réserves, en France, est également critique. Des sociétés militaires privées, viviers de personnels formés dans les unités françaises et habitués aux modes opératoires de l'armée de terre, pourraient suppléer efficacement le manque de réservistes.

Enfin, si les Nations unies se décidaient à privatiser le " Peace keeping $»^{17}$ et à recourir à des sociétés militaires privées pour des missions d'imposition et de maintien de la paix, des entreprises françaises fortes et compétentes devraient être en mesure de répondre aux appels d'offres onusiens. Elles pourraient contrebalancer le poids politique et l'agressivité commerciale des PMC anglo-saxonnes qui proposent déjà leurs services. Elles pourraient aussi contribuer à renforcer la position de la France face à un nouvel impérialisme dont les PMC ne sont que le faux nez.

\section{NOTES}

1. . Voir le site Internet de Secopex : www.secopex.com

2. . Chapleau P., Le contrat irakien de l'ancien commando de marine, Ouest-France du 14 novembre 2003.

3. . Singer P.W., Corporate Warriors : The Rise of the Privatized Military Industries, Cornell University Press, 2003, $352 \mathrm{p}$.

4. . On lira, par exemple, le livre de Bob Denard et Georges Fleury, Corsaire de la République, Robert Laffont, 1998, 437 p.

5. . Paul Barril avait été nommé à la tête des services de lutte antiterroriste par le président Patassé et avait créé la société SCPS.

6. . Les auditions sont à consulter sur le site du Réseau Voltaire (www.reseauvoltaire.net). 
7. . Pour bien découvrir ce secteur économique, consulter la revue En toute sécurité (Technopresse) qui publie un journal bimensuel et un atlas économique. Voir sur www.security-info.com

8. . Le pourcentage de pénétration du marché français par des groupes étrangers augmente. En 2002, 92\% du traitement des valeurs, 91\% du transport de fonds, $81 \%$ de la télésurveillance professionnelle, $77 \%$ des interventions sur alarme, $43 \% \mathrm{du}$ gardiennage, mais $10 \%$ seulement de la protection rapprochée, étaient confiés à des entreprises étrangères, selon l'Atlas 2003 d'En toute sécurité.

9. . Chapleau P., « Le boom de la sécurité privée en Irak », Ouest-France, 2 novembre 2003, page 2 .

10. . Des expérimentations, à Romorantin, Villacoublay et Varennes-sur-Allier, font actuellement l'objet d'un suivi par le comité directeur de l'externalisation dans l'armée de l'air (Codextaa) et par un comité de pilotage (Copextaa). Onze groupes de travail planchent aussi sur de nouvelles études exploratoires. Il s'agit en fait de généraliser l'externalisation de toutes les tâches qui ne sont pas « directement liées aux missions de préparation de combat ».

11. . Dépêche AFP du 3 novembre 2003 : Face à la controverse, l'Etat s'engage à contrôler chaque euro de la Défense.

12. . Elomari B., « DCI : privatisation de la coopération militaire française et commerce des armes », pp.70-74, in Le boom du mercenariat : défi ou fatalité, Survie, actes du colloque du 30 novembre 2000, Les documents de Damoclès, 2001, p. 70.

13. . Voir la « Tip of the Spear typology » suggérée par PW Singer dans Corporate Warriors : The Rise and Ramifications of the Privatized Military Industries, International Security, Vol.26, n³, hiver 2001/2002.

14. . La sécurité humaine est un domaine en pleine expansion, avec des hausses annuelles de l'ordre de $12 \%$ à $15 \%$, qui génère 15 milliards d'euros de chiffre d'affaires par an et qui emploie 91000 salariés (et surtout de nombreux intérimaires).

15. . La Tribune, 05-06 juillet 2001.

16. . Voir la Lettre d'information $n^{\circ} 44$ (novembre 2003) du groupe de liaison G2S : La France en Afrique : deux opérations extérieures riches d'enseignements.

17. . Un groupe de travail a été mis en place en novembre 2003 par le secrétaire général de l'ONU pour étudier un possible recours à des sociétés militaires privées. Des propositions ont été faites par des groupes privés dont l'International Peace Operations Association et le Global Security Partnership Project.

\section{INDEX}

Index géographique : France

Mots-clés : entrepreunariat, Militaires, privatisation de la sécurité, protection

Thèmes : Dyncorps, MPRI 